\title{
Atrophic dermatofibrosarcoma protuberans
}

\author{
Ola Bakry, Abdalla Attia
}

Department of Dermatology, Andrology and S.T.Ds, Faculty of Medicine, Menoufiya University, Egypt.

Corresponding author:

Dr. Ola Bakry

Department of Dermatology, Andrology and S.T.Ds, Faculty of Medicine

Faculty of Medicine Street

Shibeen El Koom, Menoufiya

Governorate, Egypt

E-mail: drola_2007@yahoo.com

\section{Key words:}

atrophic plaque, CD34, dermatofibrosarcoma protuberans, sarcoma, Moh's micrographic surgery

\begin{abstract}
Background: Dermatofibrosarcoma protuberans (DFSP) is an uncommon malignant mesenchymal tumor that arises in the dermis and is characterised by latency in its initial detection. As a rare form, atrophic or morphea-like DFSP has been documented. Atrophic DFSP resemble other benign lesions such as morphea, idiopathic atrophoderma, atrophic scar, anetoderma or lipoatrophy. It behaves like classic DFSP. It commonly favours young to middle aged adults. It has a slow infiltrative growth and a high rate of local recurrence if not completely excised. Metastases are rare and occur after repeated local recurrence. Surgical excision is the best line of treatment. Long term follow up is required to detect recurrence.
\end{abstract}

Main observations: We report a case of atrophic DFSP in a 52-year-old female patient. Diagnosis was achieved according to clinical, histopathological and immunohistochemical findings. Tumor was surgically excised with safety margin and the patient is still under follow up.

Conclusions: Atrophic DFSP is a rare variant of DFSP. It is a tumor of low to moderate grade malignancy. Surgical excision is the best line of management. Long term follow up is necessary. (J Dermatol Case Rep. 2012; 6(1): 14-17)

\section{Introduction}

Dermatofibrosarcoma protuberans is relatively uncommon soft tissue neoplasm with low to intermediate grade malignancy. Although metastasis rarely occurs, DFSP is a locally aggressive tumor with a high recurrence rate. ${ }^{1}$

Although DFSP have been reported in the literature as early as 1890, Darier and Ferrand first described it in 1924 as a distinct cutaneous disease entity called progressive and recurring dermatofibroma. ${ }^{2}$

The clinical evolution of DFSP is typified by an initial plaque stage and a later nodular stage. Rarely, the initial plaque may be atrophic or depressed and this atrophic appearance may persist. ${ }^{3}$ It has been suggested that "protuberans" could be discarded from the term DFSP altogether, as such lesions often lack the protruding nodules despite advanced growth. Atrophic DFSP, morpheaform variant of DFSP and dermatofibrosarcoma non-protuberans are synonymous terms for this atrophic clinical presentation, with atrophic DFSP being the most adopted. ${ }^{4}$

Atrophic DFSP was first reported in $1985 .^{3}$ Given the typical "protuberant" morphology of DFSP, such atrophic lesions can be difficult to diagnose clinically. Including our case, there have been 33 reported cases of this variant to date.

\section{Case Report}

A 52-year-old female presented with a well defined, atrophic, sclerotic, violaceous plaque with irregular outline, $(6 \times 5 \mathrm{~cm})$ in diameter on the left buttock (Fig. 1) of 10 years duration. Lesion was progressive and increased gradually in size since its appearance but was completely asymptomatic. Lesion had a smooth, regular surface that was depressed from the surrounding normal skin. On palpation it was not indurated and freely mobile over the underlying tissues.

Generally, the patient was well with no history of systemic diseases. Skin allover the body and mucosal surfaces were normal. Inguinal lymph nodes were not enlarged.

Clinically, lesion appeared mostly consistent with morphoea or atrophoderma. Routine laboratory investigations revealed no abnormality. Biopsy was taken after taking patient's consent. Histopathological examination of hematoxylin 


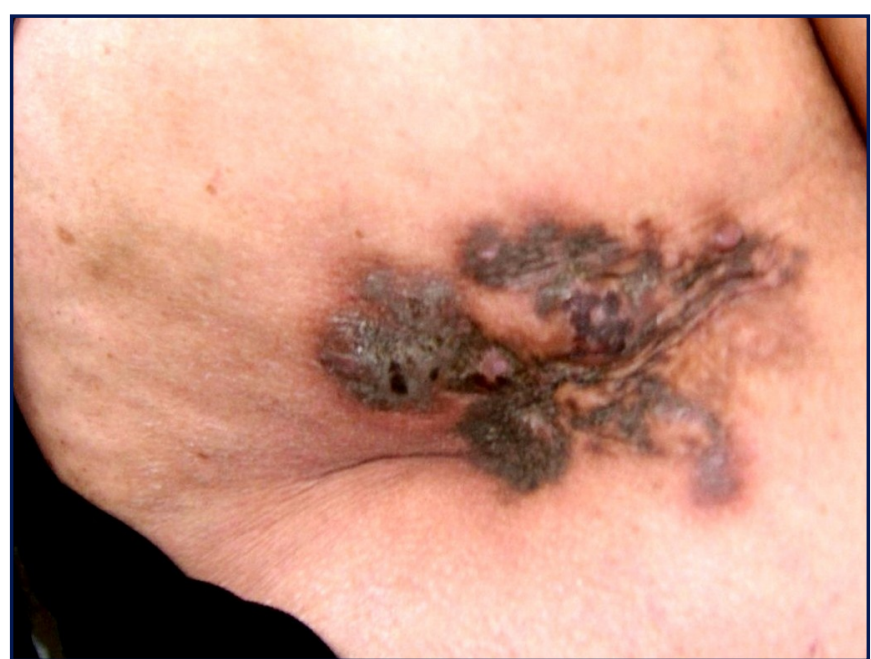

Figure 1

Violaceous atrophic well defined plaque on the left buttock with irregular outline.

and eosin-stained sections revealed normal epidermis and heavy dermal cellular infiltrate. This infiltrate is made of spindle shaped cells embeded in a fibrous stroma and arranged in a storiform pattern (Fig. 2a) that invades down to the subcutaneous tissue in a lace-like manner. The neoplastic cells showed uniform slender and elongated nuclei with scanty pale cytoplasm. No evidence of mitotic activity was detected.

The diagnosis of DFSP was made. Confirmatory immunohistochemical staining for CD34 was done and revealed positive staining of spindle cells (Fig. 2b). Lesion was removed surgically with $3 \mathrm{~cm}$ safety margin.

\section{Discussion}

DFSP is the most common type of cutaneous sarcoma. It accounts for less than $0.1 \%$ of all malignant neoplasms and approximately $1 \%$ of all soft tissue sarcomas. ${ }^{5}$

It is a very slowly growing tumor. Because of the slow progression, the diagnosis is often delayed for months to years. ${ }^{1}$

As it often shows a long-term clinical course, the very early stage of the lesions are rarely described in detail. Martin et al. ${ }^{6}$ noted that there are 4 distinguishable variants of DFSP in its early stage: (i) confluent nodular lesions forming a sclerotic plaque; (ii) keloid-like sclerotic plaque; (iii) tumor; and (iv) atrophic plaque.

The non-protuberant DFSP can be considered as the early clinical stage of DFSP before developing the typical protuberant feature or it will remain as a non prtuberant tumor, that's called atrophic DFSP. Hanabusa et al. ${ }^{7}$ reported a case of non protuberant DFSP on the cheek that was initially diagnosed as morphea and remained for 20 years before the appearance of protuberant nodules.

So, greater clinical awareness that DFSP begins and may persist as a non-protuberant atrophic plaque will allow earlier diagnosis.

While atrophic DFSP has a distinct clinical appearance, its epidemiology, histology and clinical behaviour appear to be

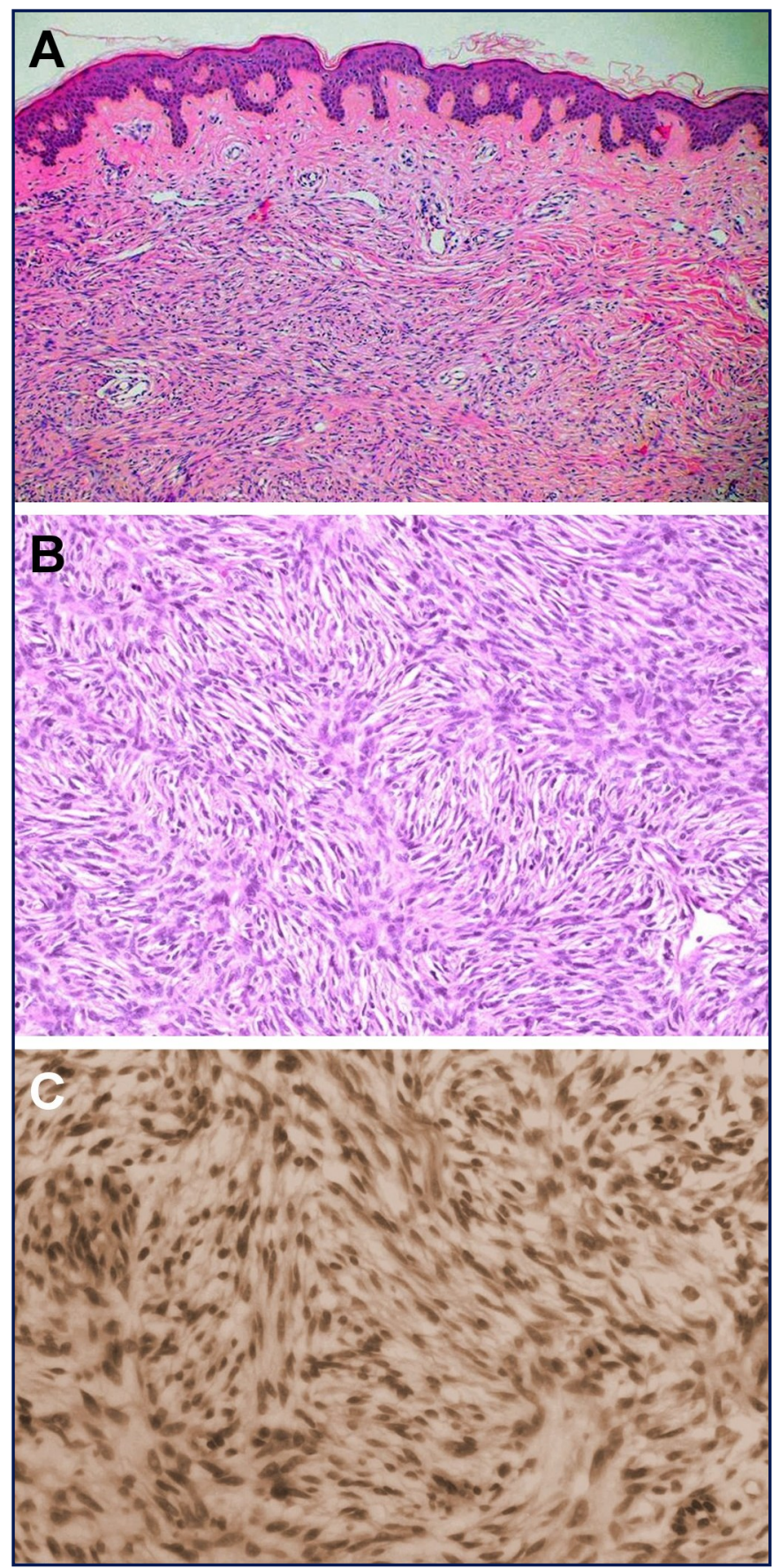

Figure 2

A) a photomicrograph showing normal epidermis. the dermis is diffusely infiltrated by spindle shaped cells embeded in a fibrous stroma and arranged in a storiform pattern (hematoxylin and eosin X100). B) Higher power magnification showing the spindle shaped neoplastic cells (hematoxylin and eosin X200) C) CD34 immunostaining showing diffuse positive staining of neoplastic cells (immunoperoxidase X200).

similar to the common protuberant type. It is most frequently diagnosed in young adults (median age 24 years). It may arise in childhood ${ }^{6}$ or be congenital. ${ }^{8}$ Men and women are similarly affected and the trunk is the most common site involved. ${ }^{4}$

This tumor is usually painless, hence easily ignored by the patient and often left untreated for many years. ${ }^{5}$ 
Mostly the tumor is mobile upon palpation; however, fixation to deeper structures such as fascia, muscle and bone may occur in late stages. ${ }^{2}$

The causes of the depressed appearance are still unknown. The marked cellularity of the dermis and infiltration of the subcutaneous fat might be the causes of the depressed presentation. ${ }^{9,10}$

The cellular origin of the tumor is not clear. Evidences support fibroblastic, histiocytic or neuroectodermal origins. ${ }^{11}$

The pathogenesis is unknown. There is no evidence of hereditary or familial predisposition. A genetic link has been found in some patients, which frequently (in more than 90\%) exhibits translocation in chromosomes 17 and $22, \mathrm{t}(17 ; 22){ }^{12}$ This rearrangement fuses the collagen type I alpha 1 (COL1A1) gene to the platelet-derived growth factor B-chain (PDGFB) gene. The resultant rearrangement causes unregulated expression of platelet-derived growth factor leading to constitutive activation of the platelet-derived growth factor receptor (PDGFR). ${ }^{13}$

Autocrine PDGFR stimulation provides antiapoptotic signals, leading to malignant transformation and growth. ${ }^{14}$

This step is believed to be a critical event in DFSP tumorigenesis. It has been postulated that abnormally truncated or assembled collagen fibres due to variable COL1A1 fragment translocation is unique to each tumour. This will lead to different stromas, and might account for the atrophic versus the typical phenotype. ${ }^{4}$

Germ line mutations of p53 have been described in patients with DFSP and breast adenocarcinoma. ${ }^{15}$ DFSP has been reported to develop after prolonged tanning-bed use. ${ }^{16}$

Histologically, atrophic DFSP is characterized by monomorphous spindle shaped cells arranged in a storiform pattern on a background of fibrous stroma. The infiltrate tends to extend to the subcutaneous fat. There is usually little nuclear pleomorphism and only low to moderate mitotic activity. The epidermis is usually spared but can be mildly atrophic. $^{1}$ The thickness of the dermis is reduced to $<50 \%$ of surrounding normal dermis, placing the subcutis close to the epidermis. ${ }^{17}$

On histopathological examination, atrophic DFSP can be mistaken for fibrohistiocytic neoplasms such as atrophic dermatofibroma, atypical fibroxanthoma and malignant fibrohistiocytoma. ${ }^{18}$

Atrophic dermatofibroma is composed of mixture of spindle shaped cells, multinucleated giant cells, inflammatory cells and hemosidrin laden histiocytes. The infiltrate does not reach the subcutaneous tissue. Malignant fibrohistiocytoma is composed of atypical and pleomorphic spindle shaped cells with frequent mitoses, multinucleated giant cells and areas of necrosis. Atypical fibroxanthoma histopathologically resemles malignant fibrohistiocytoma but polyhedral vacuolated cells are abundant, necrosis is absent and subcutaneous tissue is not involved. ${ }^{19}$

Immunohistochemical evaluation is helpful. On immunohistochemical studies, DFSP cells stain strongly with human progenitor cell antigen CD34 and are negative for factor XIIla. The latter differentiates DFSP from dermatofibroma. ${ }^{11}$

As in all cases of DFSP, no imaging studies are used in the atrophic variant unless metastatic disease is suspected. Chest radiography may be ordered for baseline screening for pulmonary metastasis in high risk cases. Computed tomography scanning is indicated if direct bone involvement or metastasis is suspected. Ultrasonography may be helpful for monitoring local tumor or regional lymph node metastasis. Reports suggest fluorodeoxyglucose (FDG) positron emission tomography scanning may be helpful in monitoring metastatic disease. ${ }^{20}$

Treatment options for atrophic DFSP are the same as classic DFSP. Moh's micrographic surgery (MMS) is the treatment of choice. Prior to MMS, surgical excision with 3 to 5 $\mathrm{cm}$ safety margin was the recommended treatment, but was associated with high rates of local recurrence. The extent of invasion is difficult to ascertain because of its ability to penetrate not only cutaneous and subcutaneous tissue, but also underlying fascia and muscle. In a three dimensional view, the tumor can be visualized as sending projections in different directions, so even a wide excision may leave residual tumor in a single or multiple foci. ${ }^{21}$

This explains the high recurrence rate of $20-50 \%$ after excision and justifies the present recommendation of wide resection with a margin of 3-5 cm.

MMS requires less tissue removal and allows complete margin assessment. However, large tumor can be a challenge for this very time consuming procedure. ${ }^{20}$

Ratner et al. ${ }^{22}$ reported that a standard wide excision with a width of $1 \mathrm{~cm}$ would leave a residual tumor in $70.7 \%$ of cases, a width of $2 \mathrm{~cm}$ would leave a residual tumor in $39.7 \%$ of cases, a width of $3 \mathrm{~cm}$ would leave a residual tumor in $15.5 \%$ of cases, and a width of $5 \mathrm{~cm}$ would leave a residual tumor in $5.2 \%$ of cases. The authors concluded that MMS is the treatment of choice for DFSP.

However, Snow et al. ${ }^{23}$ reported that nine out of 136 patients treated with MMS developed local recurrences. Of these, five patients developed recurrences even after undergoing the Moh's procedure twice.

Radiotherapy has been used as an adjuvant therapy after wide surgical excision or in those patients who have inoperable disease. Postoperative radiotherapy has been associated with a $85 \%$ cure rate. ${ }^{24}$

Imatinib, a tyrosine kinase inhibitor, has been approved to treat adult patients with unresectable, recurrent, and/or metastatic disease. Imatinib inhibits the platelet-derived growth factor receptor tyrosine kinase. ${ }^{25,26}$

Reverse transcriptase polymerase chain reaction (RT$\mathrm{PCR}$ ) and fluorescence in situ hybridization (FISH) are suggested as screening tools for the presence of COL1A1PDGFB fusion gene prior to initiation of oral imatinib molecular targeted therapy. Tumors lacking the classic $t(17,22)$ translocation mutation seem to respond poorly to imatinib. ${ }^{27}$

Although most recurrences occurred in the first 3 years after surgery, a late recurrence in the female breast has been reported. The patient experienced two delayed recurrences after 11 years and 17 years. This further underscores the importance of longterm surveillance. ${ }^{1}$ It is recommended that follow up be every 3-6 months for 3 years and then annually for life. ${ }^{28}$ 


\section{Conclusions}

We report a case of atrophic DFSP which is a rare mesenchymal tumor of low to moderate grade malignancy. It should be considered in the differential diagnoses when examining atrophic cutaneous plaque. When histopathological examination of a depressed lesion showed proliferation of spindle cells, immunohistochemical staining of CD34 is very important to make an accurate diagnosis of atrophic DFSP.

\section{References}

1. Lee SJ, Mahoney MC, Shaughnessy E. Dermatofibrosarcoma protuberans of the breast: imaging features and review of the literature. AJR Am J Roentgenol. 2009; 193: W64-69. PMID: 19542385.

2. Gloster HM Jr. Dermatofibrosarcoma protuberans. J Am Acad Dermatol. 1996; 35: 355-374. PMID: 8784271.

3. Lambert WC, Abramovits W, Gonzalez-Sevra A, Souchon E, Schwartz RA, Little WP Jr. Dermatofibrosarcoma non-protuberans: description and report of five cases of a morpheaform variant of dermatofibrosarcoma. J Surg Oncol. 1985; 28: 7-11. PMID: 2578589.

4. Young CR 3rd, Albertini MJ. Atrophic dermatofibrosarcoma protuberans: case report, review, and proposed molecular mechanisms. J Am Acad Dermatol. 2003; 49: 761-764. PMID: 14512938.

5. Criscione VD, Weinstock MA. Descriptive epidemiology of dermatofibrosarcoma protuberans in the United States, 1973 to 2002. J Am Acad Dermatol. 2007; 56: 968-973. PMID: 17141362.

6. Martin L, Combemale P, Dupin M, Chouvet B, Kanitakis J, Bouyssou-Gauthier ML, Dubreuil G, Claudy A, Grimand PS. The atrophic variant of dermatofibrosarcoma protuberans in childhood: a report of six cases. $\mathrm{Br} J$ Dermatol. 1998; 139: 719-725. PMID: 10025975.

7. Hanabusa M, Kamo R, Harada T, Ishii M. Dermatofibrosarcoma protuberans with atrophic appearance at early stage of the tumor. J Dermatol. 2007; 34: 336-339. PMID: 17408444 .

8. Marini M, Saponaro A, Magariños G, de Baldrich A, Lynch P, Remorino L. Congenital atrophic dermatofibrosarcoma protuberans. Int J Dermatol. 2001; 40: 448-450. PMID: 11679000.

9. Page EH, Assaad DM. Atrophic dermatofibroma and dermatofibrosarcoma protuberans. J Am Acad Dermatol. 1987; 17: 947-950. PMID: 3429722.

10. Fujimoto M, Kikuchi K, Okochi H, Furue M. Atrophic dermatofibrosarcoma protuberans: a case report and review of the literature. Dermatology. 1998; 196: 422-424. PMID: 9669119.

11. Ruiz-Tovar J, Fernández Guarino $M$, Reguero Callejas $M E$, Aguilera Velardo A, Arano Bermejo J, Cabañas Navarro L. Dermatofibrosarcoma protuberans: review of 20-years experience. Clin Transl Oncol. 2006; 8: 606-610. PMID: 16952850.

12. Abrams TA, Schuetze SM. Targeted therapy for dermatofibrosarcoma protuberans. Curr Oncol Rep. 2006; 8: 291296. PMID: 17254529.
13. McArthur G. Dermatofibrosarcoma protuberans: recent clinical progress. Ann Surg Oncol. 2007; 14: 2876-2886. PMID: 17647063.

14. Simon MP, Navarro M, Roux D, Pouysségur J. Structural and functional analysis of a chimeric protein COL1A1-PDGFB generated by the translocation $t(17 ; 22)(q 22 ; q 13.1)$ in Dermatofibrosarcoma protuberans (DP). Oncogene. 2001; 20: 2965-2975. PMID: 11420709.

15. Beech DJ, Long AB, Long WP. Dermatofibrosarcoma protuberans and breast cancer: genetic link or coincidental association? Am Surg. 2004; 70: 543-545. PMID: 15212412.

16. Behroozan DS, Glaich A, Goldberg LH. Dermatofibrosarcoma protuberans following tanning bed use. J Drugs Dermatol. 2005; 4: 751-754. PMID: 16302562.

17. Zelger BW, Ofner D, Zelger BG. Atrophic variants of dermatofibroma and dermatofibrosarcoma protuberans. Histopathology. 1995; 26: 519-527. PMID: 7545142.

18. Aiba S, Tabata N, Ishii H, Ootani H, Tagami H. Dermatofibrosarcoma protuberans is a unique fibrohistiocytic tumour expressing CD34. Br J Dermatol. 1992; 127: 79-84. PMID: 1382538.

19. Laskin WB. Dermatofibrosarcoma protuberans. CA Cancer J Clin. 1992; 42: 116-125. PMID: 1540852.

20. Rutgers EJ, Kroon BB, Albus-Lutter CE, Gortzak E. Dermatofibrosarcoma protuberans: treatment and prognosis. Eur J Surg Oncol. 1992; 18: 241-248. PMID: 1607035.

21. Haycox CL, Odland PB, Olbricht SM, Casey B. Dermatofibrosarcoma protuberans (DFSP): growth characteristics based on tumor modeling and a review of cases treated with Mohs micrographic surgery. Ann Plast Surg. 1997; 38: 246251. PMID: 9088462.

22. Ratner D, Thomas CO, Johnson TM, Sondak VK, Hamilton TA, Nelson BR, Swanson NA, Garcia C, Clark RE, Grande DJ. Mohs micrographic surgery for the treatment of dermatofibrosarcoma protuberans. Results of a multi-institutional series with an analysis of the extent of microscopic spread. J Am Acad Dermatol. 1997; 37: 600-613. PMID: 9344201.

23. Snow SN, Gordon EM, Larson PO, Bagheri MM, Bentz ML, Sable DB. Dermatofibrosarcoma protuberans: a report on 29 patients treated by Mohs micrographic surgery with long-term follow-up and review of the literature. Cancer. 2004; 101: 28-38. PMID: 15221986.

24. Mendenhall WM, Zlotecki RA, Scarborough MT. Dermatofibrosarcoma protuberans. Cancer. 2004; 101: 2503-2508. PMID: 15503305.

25. Rutkowski P, Wozniak A, Switaj T. Advances in molecular characterization and targeted therapy in dermatofibrosarcoma protuberans. Sarcoma. 2011; 2011: 1-6. PMID: 21559214.

26. Johnson-Jahangir H, Ratner D. Advances in the management of dermatofibrosarcoma protuberans. Dermatol Clin. 2011; 29: 191-200. PMID: 21421145.

27. Lemm D, Mügge LO, Mentzel T, Höffken K. Current treatment options in dermatofibrosarcoma protuberans. J Cancer Res Clin Oncol. 2009; 135: 653-665. PMID: 19205737.

28. Gloster HM Jr, Harris KR, Roenigk RK. A comparison between Mohs micrographic surgery and wide surgical excision for the treatment of dermatofibrosarcoma protuberans. J Am Acad Dermatol. 1996; 35: 82-87. PMID: 8682970. 